\title{
Phenotyping in COVID-19: Making the case for why science needs philosophy
}

\author{
Yi-Hern Tan ${ }^{1}{ }_{M R C P}(E d i n)$, Chee Kiang Tay ${ }^{1}{ }_{M R C P}$ (Lond)
}

COVID-19 pneumonia is caused by the severe acute respiratory syndrome coronavirus 2 (SARS-CoV-2). Among patients with SARS-CoV-2 infection, the most frequent reason for intensive care unit (ICU) admission is the development of acute respiratory distress syndrome (ARDS) and consequent hypoxaemia. ${ }^{1}$

There has been much academic discourse and debate around clinical phenotypes of COVID-19-associated ARDS (CARDS). Gattinoni et al. first described the unusual combination of profound hypoxaemia and relatively preserved lung compliance in a series of COVID-19 patients with ARDS. ${ }^{2}$ Based on distinct physiologic profiles observed in their case series, they postulated that 2 distinct phenotypes exist: type $\mathrm{L}$ that is primarily characterised by low elastance and low recruitability, and type $\mathrm{H}$ that is more akin to typical severe ARDS with high elastance and high recruitability. By attributing hypothesised differences in lung mechanics and pathophysiologic mechanisms to type L CARDS, they posited tailored treatments that ran counter to evidence-based recommendations in the management of ARDS. ${ }^{3}$ It is our view that this postulation, which became a trending topic in no time, did engender a welcomed shift in CARDS treatment from the frenetic chase for effective therapeutics to pathophysiology-based intensive care management. At this juncture, it is pertinent to highlight that the concept of phenotyping ARDS is neither a novel nor recent phenomenon. Investigators had previously identified hyperinflammatory and hypoinflammatory subphenotypes in non-COVID-19 ARDS, and the implications of this dichotomy on treatment. ${ }^{4,5}$

In this issue of the Annals, Puah et al. described a prospective cohort study that examined factors associated with mortality in 102 mechanically ventilated patients with CARDS. ${ }^{6}$ Older age, use of high-flow nasal cannula (HFNC), and acute kidney injury (AKI) and/or renal replacement therapy correlated with higher mortality. Post-intubation static compliance $\left(\mathrm{C}_{\text {stat }}\right)$ data were available for 67 patients. Using a $\mathrm{C}_{\text {stat }}$ threshold of $40 \mathrm{~mL} / \mathrm{cm} \mathrm{H} \mathrm{H}_{2} \mathrm{O}, 24(35.8 \%)$ and 43 (64.2\%) patients were classified as high and low compliance phenotypes, respectively. ${ }^{7}$ Follow-up $\mathrm{C}_{\text {stat }}$ data at 1-week post-intubation were available for 29 patients (12 and 17 patients in the high and low compliance groups, respectively). A significant reduction in $\mathrm{C}_{\text {stat }}\left(-10.5 \mathrm{~mL} / \mathrm{cm} \mathrm{H}_{2} \mathrm{O}\right.$, interquartile range -4.9 to $-18.15 ; P=0.01)$ was observed only in the high compliance group. Patients in this group also had higher mortality (33.3\% versus $11.6 \% ; P=0.03)$; this observation remained statistically significant after correcting for age, use of $\mathrm{HFNC}$ and $\mathrm{AKI}$ (odds ratio $3.5,95 \%$ confidence interval $1.1-12 ; P=0.04)$. The authors likened the high compliance phenotype in their study to previously described ARDS subtypes such as the hypoinflammatory phenotype 1 by Calfee et al. ${ }^{4}$ and focal ARDS by Constantin et al. ${ }^{8}$ However, no significant between-group differences in inflammatory biomarkers, e.g. C-reactive protein or procalcitonin were found to support this hypothesis.

The study findings suggest that CARDS patients who presented with higher $\mathrm{C}_{\text {stat }}$ that subsequently deteriorated are at a higher risk of death. However, it is imperative to evaluate the limitations and context of this study before the findings could be interpreted plainly. First, only 67 patients had post-intubation $\mathrm{C}_{\text {stat }}$ measurements, with less than half of them having a repeat measurement on day 7 after intubation. Considering that the median duration of mechanical ventilation was 11-12 days in this subgroup, it remains unclear if the marked "attrition" in numbers could be mostly accounted for by patients who had recovered quickly and adequately to undergo weaning or liberation from mechanical ventilation. Second, data such as the onset of symptoms and hypoxaemia/respiratory failure preceding intubation were unreported. As heterogeneity in CARDS (e.g. clinical trajectory, pathophysiologic mechanisms, respiratory mechanics and inflammatory profiles) is increasingly recognised, inclusion of timerelated variables could reduce their potential confounding effects on study results. ${ }^{9}$ Third, this study was conducted relatively early during the COVID-19 pandemic in Singapore. Following this study, dexamethasone and remdesivir were shown to have potentially important clinical benefits in mechanically ventilated patients with severe COVID-19 pneumonia, and are now

${ }^{1}$ Department of Respiratory \& Critical Care Medicine, Singapore General Hospital, Singapore

Correspondence: Dr Chee Kiang Tay, Department of Respiratory \& Critical Care Medicine, Singapore General Hospital, 20 College Road, Academia, Level 3, Singapore 169856.

Email: melvin.tay.c.k@singhealth.com.sg 
recommended as standard-of-care for this patient subgroup. ${ }^{10-12}$ Notwithstanding the unknown impact of these therapeutics on pulmonary physiology and mechanical ventilation, the effect is largely mitigated by the study's impressively low mortality rate of $14.7 \%$. Finally, analysis using data from the Large Observational Study to Understand the Global Impact of Severe Acute Respiratory Failure (LUNG-SAFE) study - a multinational ESICM-led prospective cohort study - revealed a wide range of respiratory system compliance $\left(\mathrm{C}_{\mathrm{rs}}\right)$ among 1,171 non-COVID ARDS patients with 1 in 8 having preserved compliance, and that lower $\mathrm{C}_{\mathrm{rs}}$ was independently associated with higher mortality. ${ }^{13}$ In other words, the so-called type L CARDS phenotype existed even before the current pandemic among patients with ARDS. Rather than a novel disparate entity, it is more appropriate to conclude that it lies on a spectrum of the same clinical syndrome, namely ARDS.

The take-home message from the evolving narrative of COVID-19 phenotyping is perhaps best encapsulated by the words, "purpose", "process" and "pragmatism". Phenotyping should have a clear purpose that is preferably actionable, e.g. an intervention that leads to improved patient-centred outcome. ${ }^{14}$ It is equally important to recognise that scientific rigour is requisite in the phenotyping process that entails among others: systematic data collection from large multicentre cohorts, robust and unbiased statistical data analysis, internal consistency measurements, and demonstration of reproducibility via prospective validation in disparate cohorts. ${ }^{14,15}$ Lastly, it is easy to be overwhelmed by an overload of new information (whether true or false) in this digital age. In philosophy, one of the core tenets in pragmatism is enquiry, which as pragmatists are persuaded, requires a presuppositionless starting-point that is only possible in the presence of doubt. Perhaps more so than ever, science needs philosophy. ${ }^{16}$

\section{REFERENCES}

1. Phua J, Weng $\mathrm{L}$, Ling $\mathrm{L}$, et al. Intensive care management of coronavirus disease 2019 (COVID-19): challenges and recommendations. Lancet Respir Med 2020;8:506-17.
2. Gattinoni L, Coppola S, Cressoni M, et al. COVID-19 Does Not Lead to a "Typical" Acute Respiratory Distress Syndrome. Am J Respir Crit Care Med 2020;201:1299-300.

3. Gattinoni L, Chiumello D, Caironi P, et al. COVID-19 pneumonia: different respiratory treatments for different phenotypes? Intensive Care Med 2020;46:1099-102.

4. Calfee CS, Delucchi K, Parsons PE, et al. Subphenotypes in acute respiratory distress syndrome: latent class analysis of data from two randomised controlled trials. Lancet Respir Med 2014; 2:611-20.

5. Famous KR, Delucchi K, Ware LB, et al. Acute Respiratory Distress Syndrome Subphenotypes Respond Differently to Randomized Fluid Management Strategy. Am J Respir Crit Care Med 2017; 195:331-8.

6. Puah SH, Cove ME, Phua J, et al. Association between lung compliance phenotypes and mortality in COVID-19 patients with acute respiratory distress syndrome. Ann Acad Med Singap 2021; 50:686-94.

7. Bos LDJ, Paulus F, Vlaar APJ, et al. Subphenotyping Acute Respiratory Distress Syndrome in Patients with COVID-19: Consequences for Ventilator Management. Ann Am Thorac Soc 2020;17:1161-3.

8. Constantin JM, Jabaudon M, Lefrant JY, et al. Personalised mechanical ventilation tailored to lung morphology versus low positive end-expiratory pressure for patients with acute respiratory distress syndrome in France (the LIVE study): a multicentre, single-blind, randomised controlled trial. Lancet Respir Med 2019;7:870-80.

9. Fan E, Beitler JR, Brochard L, et al. COVID-19-associated acute respiratory distress syndrome: is a different approach to management warranted? Lancet Respir Med 2020;8:816-21.

10. RECOVERY Collaborative Group, Horby P, Lim WS, et al. Dexamethasone in Hospitalized Patients with Covid-19. N Engl J Med 2021;384:693-704.

11. Beigel JH, Tomashek KM, Dodd LE, et al. Remdesivir for the Treatment of Covid-19 - Final Report. N Engl J Med 2020; $383: 1813-26$

12. National Institutes of Health. COVID-19 Treatment Guidelines: What's new in the Guidelines, 2021. Available at: https://www. covid19treatmentguidelines.nih.gov/about-the-guidelines/whats-new. Accessed on 11 August 2021.

13. Panwar R, Madotto F, Laffey JG, et al. Compliance Phenotypes in Early Acute Respiratory Distress Syndrome before the COVID-19 Pandemic. Am J Respir Crit Care Med 2020;202:1244-52.

14. Bos LDJ, Sinha P, Dickson RP. The perils of premature phenotyping in COVID-19: a call for caution. Eur Respir J 2020;56:2001768.

15. National Institutes of Health. Rigor and Reproducibility. Available at: https://www.nih.gov/research-training/rigor-reproducibility. Accessed on 11 August 2021.

16. Laplane L, Mantovani P, Adolphs R, et al. Opinion: Why science needs philosophy. Proc Natl Acad Sci USA 2019;116:3948-52. 